\title{
Microstructure-based numerical modeling of the solid-fluid coupling interaction in acoustic foams
}

\author{
K. Gao ${ }^{1}$, J.A.W. van Dommelen ${ }^{1}$, P. Göransson ${ }^{2}$ and M.G.D. Geers ${ }^{1}$ \\ ${ }^{1}$ Eindhoven University of Technology, Department of Mechanical Engineering, \\ Materials Technology Institute, 5600 MB Eindhoven, The Netherlands; email: \\ K.Gao@tue.nl \\ ${ }^{2}$ Marcus Wallenberg Laboratory, Department of Aeronautical and Vehicle Engineer- \\ ing, KTH Royal Institute of Technology, SE-100 44 Stockholm, Sweden
}

\section{ABSTRACT}

In this paper, based on a representative volume element (RVE) and Slattery's averaging theorem, parameters of Biot's poroelastic equations for homogenous isotropic porous materials are obtained. According to Slattery's averaging theorem, the coupling terms, which describe the inertial effects and the viscous effects, are represented by an integral of the solid-fluid interaction force. This relation provides a new approach to obtain the parameters required in Biot's equations through a direct numerical simulation of the RVE. An example of a 2D RVE is given and simulations of sound propagation in an impedance tube with a foam are conducted using Biot's equations. It is shown that the numerical coupling mass obtained from this new approach behaves qualitatively the same as an associated phenomenological model.

\section{INTRODUCTION}

During operation in a noisy environment, high-tech systems must be protected from acoustic excitations. Some porous materials, such as acoustic foams, can be used in acoustic shielding covers to improve their performance (Gentry et al., 1997), which depends on the interaction between the acoustic wave and the microstructure (Lu et al., 1999). Biot's poroelasticity theory is widely used for sound propagation problems on porous media (Allard and Atalla, 2009). It describes the poroelastic behavior of a porous material with a porosity $\phi$, which is defined as the volume fraction of the fluid. According to this theory, the equilibrium equations for an element of the porous material, which includes the solid and the fluid, are expressed as

$$
\begin{aligned}
& \rho_{11} \ddot{\boldsymbol{u}}^{S}+\rho_{12} \ddot{\boldsymbol{u}}^{F}+\tilde{b}\left(\dot{\boldsymbol{u}}^{S}-\dot{\boldsymbol{u}}^{F}\right)=\nabla \cdot \boldsymbol{\sigma}^{S} \\
& \rho_{12} \ddot{\boldsymbol{u}}^{S}+\rho_{22} \ddot{\boldsymbol{u}}^{F}+\tilde{b}\left(\dot{\boldsymbol{u}}^{F}-\dot{\boldsymbol{u}}^{S}\right)=\nabla \cdot \boldsymbol{\sigma}^{F},
\end{aligned}
$$

where $\boldsymbol{u}^{S}$ and $\boldsymbol{u}^{F}$ are the average displacements over the solid and the fluid respectively and $\boldsymbol{u}$ means the time derivative $\frac{d}{d t} \boldsymbol{u}$. It can be replaced by $j \omega \boldsymbol{u}$ for a harmonic motion, 
where $j$ is the imaginary unit and $\omega$ is the angular frequency. $\sigma^{S}$ is the product of the averaged stress prevailing in the solid and the fraction $(1-\phi) \cdot \boldsymbol{\sigma}^{F}=-\phi p_{F}{ }^{2} \mathbf{I}$ with $p_{F}$ the averaged pressure of the fluid and ${ }^{2} \mathbf{I}$ the second order unit tensor. The principle that the variation of the strain energy of the element $V$ is equal to the virtual work on the external surface $\partial V$ (Biot, 1962) shows

$$
\int_{V} \delta W d V=\int_{\partial V}\left(\left(\boldsymbol{\sigma}^{S} \cdot \boldsymbol{n}\right) \cdot \delta \boldsymbol{u}^{S}+\left(\boldsymbol{\sigma}^{F} \cdot \boldsymbol{n}\right) \cdot \delta \boldsymbol{u}^{F}\right) d A,
$$

where $\boldsymbol{n}$ is the normal vector of the surface pointing to the external domain. A linear stress-strain relation can be derived by assuming a quadratic form of the strain energy in equation (2):

$$
\begin{aligned}
& \boldsymbol{\sigma}^{S}=A \nabla \cdot \boldsymbol{u}^{S 2} \mathbf{I}+N\left(\nabla \boldsymbol{u}^{S}+\left(\nabla \boldsymbol{u}^{S}\right)^{\mathrm{T}}\right)+Q \nabla \cdot \boldsymbol{u}^{F 2} \mathbf{I} \\
& \boldsymbol{\sigma}^{F}=\left(Q \nabla \cdot \boldsymbol{u}^{S}+R \nabla \cdot \boldsymbol{u}^{F}\right)^{2} \mathbf{I} .
\end{aligned}
$$

In this stress-strain relation, the four elastic coefficients $A, N, Q$ and $R$ are expressed as

$$
\begin{aligned}
A & =\frac{(1-\phi)^{2} K_{s}-(1-\phi) K_{B}+\phi \frac{K_{s}}{K_{f}} K_{B}}{\phi \frac{K_{s}}{K_{f}}+(1-\phi)-\frac{K_{B}}{K_{s}}} \\
N & =G_{B} \\
Q & =\frac{(1-\phi) \phi K_{s}-\phi K_{B}}{\phi \frac{K_{s}}{K_{f}}+(1-\phi)-\frac{K_{B}}{K_{s}}} \\
R & =\frac{\phi^{2} K_{s}}{\phi \frac{K_{s}}{K_{f}}+(1-\phi)-\frac{K_{B}}{K_{s}}},
\end{aligned}
$$

where $K_{s}$ is the solid bulk modulus, $K_{f}$ is the effective fluid bulk modulus, and $K_{B}$ and $G_{B}$ are the bulk and the shear modulus of the porous material in vacuum. Furthermore, $\tilde{b}$ is the viscous coefficient. The density coefficients $\rho_{11}, \rho_{12}$ and $\rho_{22}$ are related to the solid density $\rho_{s}$ and the fluid density $\rho_{f}$

$$
\begin{aligned}
& \rho_{11}=(1-\phi) \rho_{s}+\rho_{a} \\
& \rho_{12}=-\rho_{a} \\
& \rho_{22}=\phi \rho_{f}+\rho_{a} .
\end{aligned}
$$

Here, $\rho_{a}$ is an added mass representing the solid-fluid kinetic coupling effects (Biot, 1962).

In equations (1), the solid-fluid coupling effects appear as $\rho_{a}$ and $\tilde{b}$. Johnson et al. studied the fluid motion in rigid porous media and proposed an expression of the dynamic tortuosity based on the limiting behaviors of high and low frequencies (Johnson et al., 1987). According to their result, the viscous coefficient is written as

$$
\tilde{b}=\frac{\mu_{f} \phi^{2}}{k_{0}} \sqrt{1+\frac{j \omega \rho_{f}}{\mu_{f}}\left(\frac{2 \alpha_{\infty} k_{0}}{\Lambda \phi}\right)^{2}},
$$


where $\mu_{f}$ is the dynamic viscosity of the fluid, $k_{0}$ is the static permeability. $\alpha_{\infty}$ is the tortuosity for the case of the same microstructure saturated with an ideal fluid. $\Lambda$ is the viscous characteristic length introduced by Johnson et al. and is a ratio of the solidfluid interface and the pore volume with a weight function of the local fluid-velocity field (Johnson et al., 1987). Besides, the added mass $\rho_{a}$ is

$$
\rho_{a}=\phi \rho_{f}\left(\alpha_{\infty}-1\right)
$$

The tortuosity, the viscous characteristic length and the static permeability can be obtained through experimental measurements (Leclaire et al., 1996) or numerical calculations (Perrot et al., 2012). Johnson et al.'s phenomenological model shows a good agreement with experiments in some cases (Johnson et al., 1987; Champoux and Allard, 1991). However, this model does not include the influence of the solid mechanical properties and the viscous characteristic length is difficult to measure accurately through experiments because it requires the microscopic interface velocity of the fluid.

This paper focuses on obtaining the added mass and the viscous coefficient in Biot's equations from the homogenized behavior of a representative volume element (RVE). A micro-macro relation is obtained based on Slattery's averaging theorem. Thereafter, a numerical simulation of a $2 \mathrm{D} \mathrm{RVE}$ is discussed.

\section{MICRO-MACRO RELATION}

In this section, Slattery's averaging theorem is used to obtain a micro-macro relation. For brevity, only the main results are presented and detailed derivations can be found in (Gao et al.). It is assumed that a macroscopic point in a porous material can be represented by a microscopic RVE. In order to distinguish two types of properties, the macroscopic quantities are all denoted by the uppercase letters $\mathrm{S}$ and $\mathrm{F}$; the microscopic ones are denoted by the lowercase letters $\mathrm{s}$ and $\mathrm{f}$. It should be noted that the motion is assumed to be harmonic.

Based on the general transport theorem, Slattery's averaging theorem discusses the derivative of an integral over a variable domain for porous media (Slattery, 1967). This theorem gives the expression of the gradient of an integral of a phase property $\psi$ in porous media over the phase volume $V_{\xi}$ :

$$
\nabla \int_{V_{\xi}} \psi d V=\int_{S_{e}^{\xi}} \psi \boldsymbol{n} d A
$$

where $\boldsymbol{n}$ is the outward normal vector of the external surface $S_{e}^{\xi}$ of the phase $\xi$. This theorem is also valid for tensorial properties.

According to Slattery's theorem, a relation between the macroscopic properties in equations (1) and the microscopic properties of the RVE can be set up. By assuming that a macroscopic property is a bulk or phase average of the associated microscopic 
property, equations (1) can be rewritten as

$$
\begin{aligned}
-\omega^{2}(1-\phi) \rho_{s} \overline{\boldsymbol{u}^{s}} & =(1-\phi) \nabla \cdot \overline{\boldsymbol{\sigma}^{s}}-\left(\omega^{2} \rho_{a}-j \omega \tilde{b}\right)\left(\overline{\boldsymbol{u}^{f}}-\overline{\boldsymbol{u}^{s}}\right) \\
-\omega^{2} \phi \rho_{f} \overline{\boldsymbol{u}^{f}} & =\phi \nabla \cdot \overline{\boldsymbol{\sigma}^{f}}+\left(\omega^{2} \rho_{a}-j \omega \tilde{b}\right)\left(\overline{\boldsymbol{u}^{f}}-\overline{\boldsymbol{u}^{s}}\right)
\end{aligned}
$$

where the phase average $\overline{\boldsymbol{A}^{\xi}}$ is defined by

$$
\overline{\boldsymbol{A}^{\xi}}=\frac{1}{V_{\xi}} \int_{V_{\xi}} \boldsymbol{A}^{\xi} d V .
$$

Then, by comparing equations (9) with the corresponding bulk averages of the linearized microscopic governing equations of the fluid and the solid, a micro-macro relation is obtained:

$$
\frac{1}{V} \int_{S_{i}} \boldsymbol{n} \cdot \boldsymbol{\sigma}^{f} d A=\omega^{2}\left(\rho_{a}+\frac{\tilde{b}}{j \omega}\right)\left(\overline{\boldsymbol{u}^{f}}-\overline{\boldsymbol{u}^{s}}\right) .
$$

Here, the normal vector $\boldsymbol{n}$ on the interface is pointing from the fluid to the solid.

In Biot's poroelastic equations, the added mass $\rho_{a}$ and the viscous coefficient $\tilde{b}$ give a global description of the inertial and viscous coupling effects between the fluid and the solid. Therefore, the sum $\rho_{a}+\frac{\tilde{b}}{j \omega}$ is referred to as the coupling mass in this paper. Because equation (11) shows the relation of the coupling mass and the microscopic quantities, which can be solved by a direct numerical simulation of the RVE with the corresponding microscopic governing equations, the coupling mass $\rho_{a}+\frac{\tilde{b}}{j \omega}$ can be obtained according to this micro-macro relation.

Practically, the calculation of the surface integral on the fluid-solid interface in equation (11) may be difficult. According to Slattery's theorem and the divergence theorem, the micro-macro relation can also be changed to:

$$
-\omega^{2} \phi \rho_{f} \overline{\boldsymbol{u}^{f}}-\frac{1}{V} \int_{S_{e}^{f}} \boldsymbol{n} \cdot \boldsymbol{\sigma}^{f} d A=\omega^{2}\left(\rho_{a}+\frac{\tilde{b}}{j \omega}\right)\left(\overline{\boldsymbol{u}^{f}}-\overline{\boldsymbol{u}^{s}}\right) .
$$

This equation is more practical to use in numerical simulations because the force on an external boundary of an RVE is given in both a force and a displacement boundary conditions.

\section{NUMERICAL RESULT}

Based on the micro-macro relation in equation (11) or (12), the coupling mass can be obtained from a direct numerical simulation of the RVE. In order to evaluate this new approach, a 2D RVE is simulated and the calculated coupling mass is compared with the Johnson et al. model. Then, these parameters are applied in Biot's equations to solve a sound propagation problem in an impedance tube. 
As shown in figure 1, a 2D RVE is used that is based on an analysis of a realistic $92 \%$ porosity 40 ppi Duocel open-cell aluminum foam (Perrot et al., 2008). In figure 1, the distance between two circles is $884 \mu \mathrm{m}$ and the radius $161 \mu \mathrm{m}$. The geometry data is calculated from the porosity and a 3D $\mu \mathrm{CT}$ analysis (Perrot et al., 2008). The fluid in the simulation is air. While the air is compressible in reality, an incompressible flow model may still be a good approximation under the small deformation condition. In order to check this, both a compressible flow model and an incompressible flow model (Kundu et al., 2011) are used. The compressible flow model is derived based on the ideal gas state equation and the isothermal condition. A periodic boundary condition is applied horizontally (i.e. along the left and right boundaries) and a symmetric boundary condition is used for the top and bottom boundaries. Moreover a constant pressure difference is applied between the left and the right boundaries.

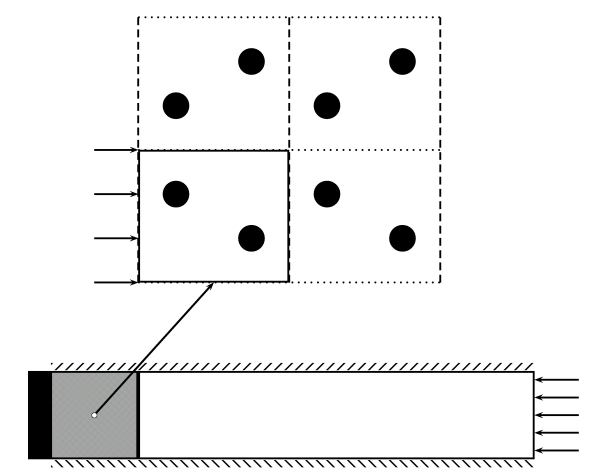

Figure 1. 2D RVE of the porous material in the impedance tube. The black solid circles are the solid phase. The RVE is enclosed by the solid black frame. The arrows represent the pressure.

The numerical coupling mass is obtained by equation (12). For comparison, the Johnson et al. model is also used to obtain the coupling mass according to equations (6) and (7). The required parameters $\left(\alpha_{\infty}, \Lambda\right.$ and $\left.k_{0}\right)$ are evaluated according to the numerical method in reference (Perrot et al., 2008) and they are given in table 1 . The results of the coupling mass $\rho_{a}+\tilde{b} /(j \omega)$ are plotted with respect to the frequency in figure 2 . The numerical results of the two fluid models (i.e. compressible and incompressible) are very close, implying that it is acceptable to use an incompressible flow model to simulate the fluid motion in the RVE. Furthermore, it is observed that the numerical coupling mass behaves qualitatively the same as the Johnson et al. model. Quantitatively, the imaginary parts are close, but the difference of the real parts is significant. According to equation (6), the second of term of the coupling mass $\left(\frac{\tilde{b}}{j \omega}\right)$ becomes zero when the frequency is very high. The high-frequency difference between the two approaches in figure 2 suggests that equation (12) gives a higher added mass compared to the Johnson et al. model.

With the obtained coupling mass, Biot's equations can be applied to sound propagation problems in porous media. As shown in figure 1, an impedance tube with the 
Table 1. Parameters for the Johnson et al. model.

\begin{tabular}{lll} 
Material property & Symbol & Value \\
\hline Tortuosity & $\alpha_{\infty}$ & 1.08 \\
Viscous characteristic length & $\Lambda$ & $9.98 \times 10^{-4} \mathrm{~m}$ \\
Static permeability & $k_{0}$ & $4.43 \times 10^{-8} \mathrm{~m}^{2}$ \\
\hline
\end{tabular}

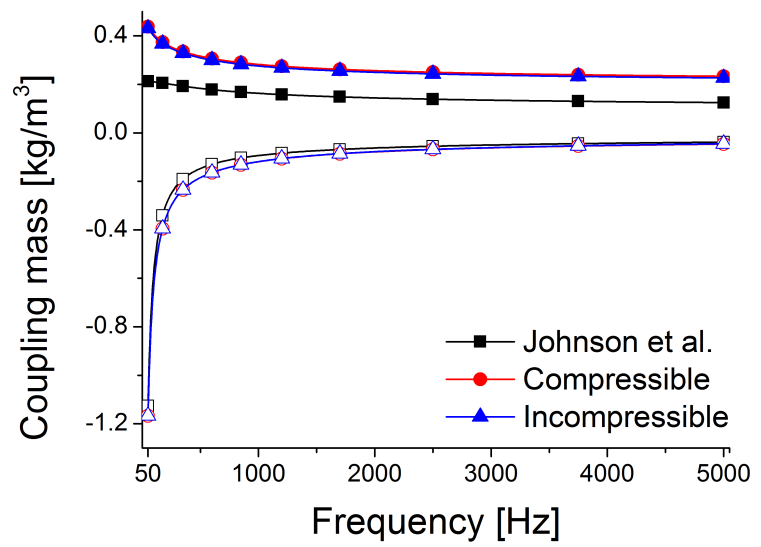

Figure 2. Coupling masses of various methods. The curves with solid symbols are the real part of the coupling mass and the curves with open symbols are the corresponding imaginary parts.

aluminum foam is simulated. The effective bulk modulus for the air is calculated according to reference (Champoux and Allard, 1991) and the other mechanical properties required in equation (4) are given in table 2. Because the air does not contribute to the shear modulus of the foam (Allard and Atalla, 2009) and this foam is made of aluminum, the properties of the foam in air are just the ones in vacuum. The poroelasticacoustic coupling condition in reference (Debergue et al., 1999) is adopted on the interface of the foam and the air. The upper and the lower boundaries of the tube are set as sound soft walls, where the gradient of the pressure is zero. The left boundary of the foam is a sound hard wall, presenting a rigid soundproofing wall. Sound absorption coefficients are calculated according to the transfer function method (ISO 10534-2:1998) and the results are shown in figure 3 . It shows that the sound absorption coefficient becomes higher for the numerical coupling mass, suggesting that more energy is dissipated in the case of a larger coupling mass.

\section{CONCLUSION}

In this paper, based on the homogenized behavior of an RVE, a new approach is developed in order to obtain the parameters determining the solid-fluid coupling effects in Biot's poroelastic equations for homogenous isotropic porous materials. This new 
Table 2. Mechanical properties for the simulation of the impedance tube.

\begin{tabular}{lll} 
Material property & Symbol & Value \\
\hline Bulk modulus for the aluminum & $K_{s}$ & $76 \mathrm{GPa}$ \\
Bulk modulus for the foam & $K_{B}$ & $100 \mathrm{MPa}$ \\
Shear modulus for the foam & $G_{B}$ & $200 \mathrm{MPa}$ \\
\hline
\end{tabular}

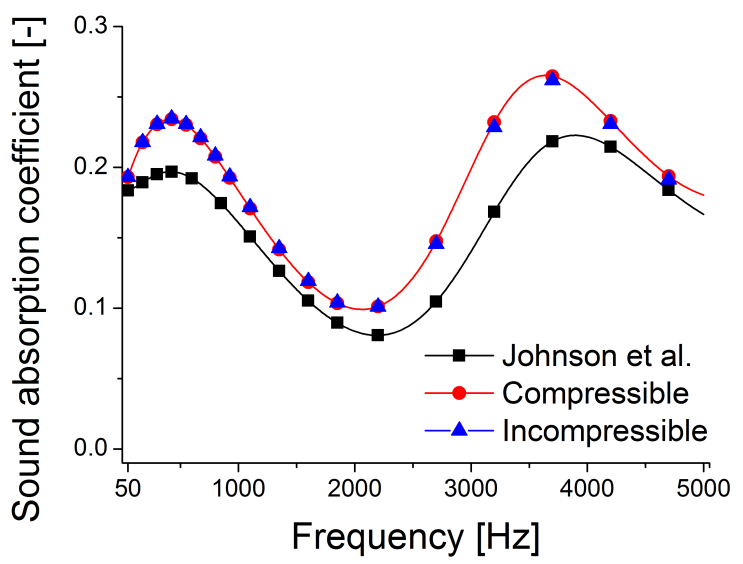

Figure 3. Sound absorption coefficients by using Biot's equations with the coupling masses in figure 2.

approach, which also considers the microstructure of the porous materials, does not require phenomenological models involving the viscous characteristic length such as the Johnson et al. model. The numerical result of the 2D RVE shows that the numerical coupling mass calculated from this new approach behaves qualitatively the same as the phenomenological model. However, the added mass in this approach seems to be larger than the one of the phenomenological model. Furthermore, the sound propagation problem in the impedance tube is simulated by using Biot's equations with these obtained parameters. It shows that a larger coupling mass leads to a higher sound absorption coefficient.

\section{REFERENCES}

Allard, J. F. and Atalla, N. (2009). Propagation of Sound in Porous Media: Modelling Sound Absorbing Materials, 2nd. John Wiley and Sons Ltd.

Biot, M. A. (1962). "Mechanics of deformation and acoustic propagation in porous media.” Journal of Applied Physics, 33(4), 1482-1498.

Champoux, Y. and Allard, J. F. (1991). "Dynamic tortuosity and bulk modulus in airsaturated porous media.” Journal of Applied Physics, 70(4), 1975-1979.

Debergue, P., Panneton, R., and Atalla, N. (1999). "Boundary conditions for the weak 
formulation of the mixed (u,p) poroelasticity problem." Journal of Acoustical Society of America, 106(5), 2383-2390.

Gao, K., van Dommelen, J. A. W., Göransson, P., and Geers, M. G. D. "Microstructurebased numerical modeling of the solid-fluid coupling interaction in acoustic foams." In preparation.

Gentry, C. A., Guigou, C., and Fuller, C. R. (1997). "Smart foam for applications in passive-active noise radiation control." Journal of Acoustical Society of America, 101(4), 1771-1778.

Johnson, D. L., Koplik, J., and Dashen, R. (1987). "Theory of dynamic permeability and tortuosity in fluid-saturated porous media." Journal of Fluid Mechanics, 176, 379-402.

Kundu, P. K., Cohen, I. M., and Dowling, D. R. (2011). Fluid Mechanics, Fifth Edition. Academic Press.

Leclaire, P., Kelders, L., Lauriks, W., Melon, M., Brown, N., and Castagnéde, B. (1996). "Determination of the viscous and thermal characteristic lengths of plastic foams by ultrasonic measturements in helium and air." Journal of Applied Physics, 80(4), 2009-2012.

Lu, T. J., Hess, A., and Ashby, M. F. (1999). "Sound absorption in metallic foams." Journal of Applied Physics, 85(11), 7528-7539.

Perrot, C., Chevillotte, F., and Panneton, R. (2008). "Dynamic viscous permeability of an open-cell aluminum foam: Computations versus experiments." Journal of Applied Physics, 103(024909).

Perrot, C., Chevillotte, F., Hoang, M. T., Bonnet, G., Bécot, F.-X., Gautron, L., and Duval, A. (2012). "Microstructure, transport, and acoustic properties of opencell foam samples: Experiments and three-dimensional numerical simulations." Journal of Applied Physics, 111(014911).

Slattery, J. C. (1967). "Flow of viscoelastic fluids through porous media." American Institute of Chemical Engineers Journal, 13(6), 1066-1071. 